\title{
Prognostic Indexes for Alcoholic Hepatitis \\ REVIEW
}

\section{Abstract}

Alcohol abuse is the major source of liver disease. The prevalence of alcoholic hepatitis (AH) is unknown, but histologic studies demonstrated that $\mathrm{AH}$ may be present in approximately $10 \%$ to $35 \%$ of hospitalized patients with alcoholism. The assessment of severity permits the identification of patients that will improve without medical therapy and those that will have a high mortality if not treated. A variety of scoring systems has been used to quantity the severity of $\mathrm{AH}$ and guides its treatment. The scores more commonly used are: Maddrey's discriminant function (DF), Model for End-Stage Liver Disease (MELD), Glasgow Alcoholic Hepatitis Score (GAHS) and Age, serum Bilirubin, INR (International Normalized Ratio), and serum Creatinine (ABIC). Some others prognostic indexes assess the efficacy of treatment, like Lille score, and Early Change in Bilirubin Levels. Histologic findings are showed to predict additional findings like risk of infection and poor prognosis even in a subgroup considered of better prognosis. The authors strongly recommend liver biopsy to confirm the diagnosis of $\mathrm{AH}$ and to discriminate patients with risk of infection and death without medical therapy. In our point of view, this method has been showed to be the best prognostic markers for $\mathrm{AH}$ in nowadays. In conclusion, $\mathrm{AH}$ is a severe complication among heavy drinkers and frequently results in poor short term prognosis. Various clinical scores are useful to differentiate patients with high mortality if not treated and are similar in predicting the outcome. More recently, the liver biopsy and a histologic score including fibrosis, megamitochondria, neutrophil infiltration and bilirubinostasis showed promising results and should be recommended.
Gabriela P. Coral', Cristiane V. Tovo', Mariana de A. Pranke', Angelo A. de Mattos ${ }^{1}$

1 Department of Gastroenterology; Universidade Federal de Ciências da Saúde de Porto Alegre (UFCSPA); Porto Alegre - RS; Brasil.

\section{Contact information:}

\section{Gabriela P Coral.}

Address: Rua Padre Chagas 185/505, Porto Alegre, 90570-080, Brasil.

Tel: $+55-51-32148158$.

Fax: $+55-51-32148238$.

$\risingdotseq$ g.coral@terra.com.br

\author{
Keywords \\ Alcoholic Hepatitis; Prognostic \\ Indexes; Liver Biopsy; \\ Steatosis; Alcoholism.
}

\section{Introduction}

Global Disease Burden was evaluated in a recent study, showing that alcohol use was the third leading source of disease. Importantly, this study considered 67 risk factors at 21 regions of the world [1]. Mo- 
reover, alcohol use is the major source of liver disease worldwide and is responsible for about 2.5 million deaths annually $[2,3]$.A study showed that alcoholic liver cirrhosis was the cause of 493,300 deaths and 14,544,000 disability adjusted life years, indicating $0.9 \%$ of all global deaths, $47.9 \%$ of all liver cirrhosis deaths and 80,600 deaths of liver cancer (14,800 female deaths and 65,900 male deaths) in 2010 [4].

In a population-based study evaluating 16 countries of America the mortality of alcohol was also important. Liver disease was responsible for $63 \%$ of deaths following by mortality for neuropsychiatric disorders [5].

In Brazilian population, liver diseases were the eighth leading cause of death. Cirrhosis was the main etiology of death by liver disease in Brazil and alcohol was the second leading cause of cirrhosis [6].

Fatty liver develops in approximately $90 \%$ of individuals who drink more than $60 \mathrm{~g} /$ day of alcohol and continuous alcohol use (40 g/day) increased the risk of progression to fibrosis and cirrhosis in approximately $37 \%[7,8]$. Some patients with Alcoholic Liver Disease (ALD) will develop alcoholic hepatitis $(\mathrm{AH})$, which is a clinical syndrome of liver injury that frequently occurs after decades of heavy alcohol use and has a significantly poor prognosis $[9,10]$. AH can range from mild to severe, lifethreatening, and can present acutely or in a patient with chronic ALD $[2,10]$. The true prevalence of $\mathrm{AH}$ is unknown, but histologic studies of patients with ALD demonstrated that $\mathrm{AH}$ may be present in approximately $10 \%$ to $35 \%$ of hospitalized patients with alcoholism [2, 11].Clinically, the most frequent presentation of $\mathrm{AH}$ is a rapid onset of jaundice; other common signs and symptoms are fever, ascites and encephalopathy [10]. Moreover, some patients with cirrhosis can have an acute decompensation named acute-on-chronic liver failure (ACLF), a condition associated with multiple organ failure and higher mortality. Recently, a multicen- ter European study, evaluating 1,343 hospitalized patients with cirrhosis and acute decompensation, showed a 28-day mortality of $33.9 \%$ among patients with ACLF and $1.9 \%$ among those who did not have this complication. In this study, the leading cause of ACLF were alcoholic cirrhosis [12].

Traditionally, corticosteroids are recommended as first line therapy for severe $\mathrm{AH}$, although some studies presented contradictory results $[2,13,14]$. Three of five meta-analyses showed improvement in short-term survival in $\mathrm{AH}$ patients receiving corticosteroid treatment $[15,16,17]$, whereas two metaanalyses showed no improvement [18].

Pentoxifylline has also been used in patients with severe $\mathrm{AH}$. Three randomized clinical trials compared pentoxifylline in combination with corticosteroids and corticosteroids monotherapy in severe $A H[19,20,21]$. In these studies there was no additional survival for the combination of drugs compared with corticosteroids alone in a 6-month survival. The guidelines of AASLD recommend pentoxifylline in the patients with severe $\mathrm{AH}$, especially if there are contraindications to the corticosteroids [2]. The EASL guidelines recommend using pentoxifylline instead of corticosteroids in patients with sepsis [14].

A recent large trial randomized 1103 patients with severe $\mathrm{AH}$ to one of four treatment groups: prednisolone and pentoxifylline, prednisolone and placebo, pentoxifylline and placebo, or double placebo. Prednisolone, but not pentoxifylline, was associated with a lower risk for 28-day mortality [22].

Newly, the addiction of metadoxine to standard treatment with corticosteroids in patients with severe $\mathrm{AH}$ was evaluated. Seventy patients with severe disease were randomized to receive prednisone or prednisone plus metadoxine. Patients treated with metadoxine had a higher survival at 30 days $(74.3 \%$ vs. $45.7 \%$ ) and at 90 days (68.6\% vs. $20.0 \%$ ). Additionally, patients treated with the combination of drugs had fewer encephalopathy and hepatorenal syndrome, severe complications of $\mathrm{AH}$ [23]. On the 
other hand, further studies were necessary to confirm these results.

Mild AH often resolves with abstinence of alcohol while the severe form has worse prognosis; therefore, the assessment of severity permits the identification of patients that will improve without medical therapy and those that will have a high mortality if not treated [24].

A variety of scoring systems has been used to quantity the severity of $\mathrm{AH}$ and guides its treatment. The scores more commonly used are: Maddrey's discriminant function (DF), Model for End-Stage Liver Disease (MELD), Glasgow Alcoholic Hepatitis Score (GAHS) and Age, serum Bilirubin, INR (International Normalized Ratio), and serum Creatinine (ABIC) [25-32]. Some others prognostic indexes assess the efficacy of treatment, like Lille score, and Early Change in Bilirubin Levels [33-34].

\section{Classic Prognostic Scores}

The first and the most frequent score used to identify patients with poor prognosis in $\mathrm{AH}$ is Maddrey's DF. This score was first describing in a placebocontrolled study aimed to assess the efficacy of corticosteroid therapy in 55 patients with $\mathrm{AH}$. The original formula considered the absolute value of prothrombin time (PT) [35]. Further, the authors presented a modification in the original score (DF) using prolongation of PT in seconds (over control) instead of the absolute value, as follows: $4.6 \times(\mathrm{PT}$ - control time) + serum bilirubin ( $\mathrm{mg} / \mathrm{dL})$, in a randomized multicenter trial with methylprednisolone therapy in patients with severe $\mathrm{AH}$. In this trial, patients with a score $\geq 32$ in a placebo group had a mortality of $35 \%$ in 28 days [25]. Subsequently to this study, DF is the prognostic marker of $\mathrm{AH}$ more frequently used.

The MELD score, traditionally used for the prediction of mortality from end-stage liver disease, has also been used as a prognostic marker in alcoholic hepatitis [26-29]. Sheth et al [27] studied 34 patients with severe $\mathrm{AH}$ and demonstrated that a MELD score higher than 11 or the presence of ascites and bilirubin over than $8 \mathrm{mg} / \mathrm{dl}$ was an indicative of poor prognosis. In the same manner, a retrospective study of 73 patients with $\mathrm{AH}$ demonstrated that MELD was the only independent predictor of mortality; MELD score of 21 had a sensitivity of $75 \%$ and a specificity of $75 \%$ in predicting mortality in 90 days [28]. The accuracy of MELD score in different moments was also evaluated in 202 patients with $\mathrm{AH}$. In this study, MELD score at admission, MELD score at first week, and first week increase in MELD score were independently associated with in-hospital mortality. Furthermore, when MELD score at first week was equal or higher than 20, the sensitivity (91\%) and specificity $(85 \%)$ to predict mortality was superior than MELD score at admission or first week change in MELD score [29].

The inclusion of sodium in MELD score has also been evaluated. A prospective study from Mayo Clinic showed that MELD score of 27 and a MELD Na score of 28 had sensitivity of $76.5 \%$ and $87.5 \%$ and specificity of $64.9 \%$ and $52.5 \%$, to predict mortality in $\mathrm{AH}$ respectively. Furthermore, in patients with $\mathrm{AH}$ and ascites, MELDNa was a better predictor of mortality in 180 days than MELD [36].

Other score that is traditionally used for the assessment of $\mathrm{AH}$ prognosis is the GAHS. Forrest et al [30] analyzed the variables related to poor prognosis of $\mathrm{AH}$ in 241 patients. The variables identified (age, white blood cell count, urea, PT and bilirubin) were included in GAHS score and validated in a distinct cohort of 195 patients. Patients with AH and GAHS greater than 9 had a poor prognosis. Moreover, in this study, GAHS was more accurate than DF in predicting outcome at 28 and 84 days after admission [30]. Further, the same authors evaluated the efficacy of GAHS to identify patients who would benefit from corticosteroids. They select 225 patients with an DF greater than or equal to 32 in a multicenter study; of these, 144 patients also had a GAHS greater than or equal to 9. When GAHS 
was less than 9, there was no difference in survival between patients untreated or treated with corticosteroids. On the other hand, when patients with a GAHS greater than or equal to 9 were evaluated, it was demonstrated that those treated had a higher survival in 28 days comparing with untreated $(78 \%$ and $52 \%$ respectively). Moreover, they showed that even in patients with DF greater than or equal to 32, when GAHS was less than 9 and, there was no significant benefit from treatment with corticosteroids [31].

Dominguez et al [32] analyzing 103 patients with $\mathrm{AH}$, demonstrated that age, serum bilirubin, INR and serum creatinine were independently associated with mortality in 90 days of admission for $\mathrm{AH}$. According to these results, the authors created the Age, serum Bilirubin, INR, and serum Creatinine $(A B I C)$ score: (age $\times 0.1)+($ serum bilirubin $\times 0.08)+$ (serum creatinine $\times 0.3)+($ INR $\times 0.8)$. Patients with low, intermediate, and high risk of death at 90 days (100\%, $70 \%$, and $25 \%$ of survival rate, respectively) were identified [32].

Some scores aimed to evaluating the response of corticosteroid therapy. Lille Score is a dynamic model that estimate the response to this therapy in the day seven of therapy; six months survival was $25 \%$ in patients with Lille score $>0.45$ and $85 \%$ in those with Lille score $<0.45$ [33]. In the same manner, studies demonstrated that any fall in serum bilirubin level after 1 week of corticosteroid therapy (Early Change in Bilirubin Levels) or more specifically a $25 \%$ fall can be simple indicators of corticosteroid response [34, 37].

Recently, because of the heterogeneity of previous studies, some authors attempted to compare the classic scores. In this setting, Sandahl et al [38] evaluated and compare GAHS, MELD, MELDNa, Lille model and $\mathrm{ABIC}$ in 274 patients demonstrating similar results between the scores in predicting mortality in $\mathrm{AH}$ at day 28, 84 and 180 [38].

Similarly, Palaniyappan et al [39] compared five scoring systems: Child Pugh, MELD, DF, GAHS and
$\mathrm{ABIC}$ score in 44 patients with $\mathrm{AH}$ confirmed by histology. This study showed that with exception of Child Pugh, that presented worse results, the other scores have a good comparable accuracy in predicting survival at 30 days, 90 days, 6 months, and 1 year. Nevertheless, they are equally poor in predicting survival in 6 months and 1 year (ROC curve not exceeding $0.74 \%$ and $0.66 \%$ respectively) [39].

Another prospective study analyzed the ability of GAHS, DF, MELD and ABIC in predicting mortality in $\mathrm{AH}$ and didn't find any difference between the scores in 182 patients at 28 and 90 days [40].

More recently, Papastergiou et al [41] aimed to validate the following nine score systems: DF; GAHS; MELD; ABIC; MELDNa; Early Change in Bilirubin Levels (ECBL); United Kington End-stage Liver Disease (UKELD); a 25\% fall in bilirubin during the treatment and Lille score. In this study, the authors evaluated 71 patients with $\mathrm{AH}$ (confirmed by biopsy) and compared the prognostic models with respect to mortality in 30 and 90 days [41]. The results revealed similar areas under the receiver operating characteristics curve (AUROCs) for all scores. Additionally, the models showed very good negative predictive values (86-100\%), signifying that these scores are best to recognize patients with low risk of mortality.

To validate the prognosis of $\mathrm{AH}$ in a US population, Cuthbert et al [42] studied 148 patients and analyzed a revised DF (using INR instead of PT) and peak values of MELD. The authors showed that the most significant predictor of short-term mortality was peak values of MELD, followed by revised DF [42].

Prognostic model of Asian patient cohort (MAGIC) were also lately presented and includes the following parameters: bilirubin, prothrombin time, creatinine, potassium at admission, and a spontaneous change in bilirubin levels from day 0 to day 7. MAGIC was able to accurately predict mortality in Asian patients with severe $\mathrm{AH}$ [43]. 


\section{Liver Histology as Prognostic Factor}

Alcoholic steatohepatitis is histologically characterized by steatosis, ballooning degeneration of hepatocytes, and lobular infiltration by inflammatory cells, particularly polymorphonuclear cells [44]. Additional findings include the presence of megamitochondria and Mallory bodies. Periportal ductular proliferation, ductular bilirubinostasis, and intraparenchymal cholestasis may also be observed. Fibrous tissue classically localizes in central zone (zone 3), in the terminal hepatic vein and in perisinusoidal and pericellular areas, frequently resulting in the typical "chicken-wire fibrosis" [45, 46].

Liver biopsy can be necessary to confirm $\mathrm{AH}$ and it's recommended in American Association for the Study of Liver Diseases (AASLD) guidelines however it's not mandatory [2]. The last guideline of European Association for the Study of Liver (EASL) also recommends liver biopsy, but only in patients with a high risk of dying without pharmacotherapy [14]. On the other hand, some of these patients have a contraindication to percutaneous liver biopsy, frequently they patients have ascites and clotting abnormalities; transjugular biopsy is recommended in these cases [24].

Spahr et al [47] assessed the liver biopsy of 163 patients with $\mathrm{AH}$ and demonstrated that intraparenchymal cholestasis was more prevalent in non survivors compared to survivors (70\% vs $25 \%$ ) at multivariate analysis. Moreover, serum bilirubin was higher in patients with severe intraparenchymal cholestasis [47].

Recently, Altamirano et al [48] identified four histological findings (fibrosis, neutrophil infiltration, type of bilirubinostasis, and presence of megamitochondria) associated with mortality at day 90 in $\mathrm{AH}$ [48]. This histologic features were further graded and classified as Alcoholic Hepatitis Histological Score (AHHS) and presented as low (0-3 points), moderate (4-5 points), or high (6-9 points) according to the risk of mortality in 90 days: $3 \%, 19 \%$ and $51 \%$, respectively. In addition, in patients treated with corticosteroids, the three months survival was $85 \%$ when AHHS score less than 5 and only $51 \%$ when the score was equal or higher than 5. Interesting, when analyzing patients with a MELD score less than 21, a score that traditionally is associated with a better outcome [28], the AHHS was also able to discriminate two different rates of survival, $94 \%$ in patients with AHHS less than 5 vs $72 \%$ in those with score equal or higher than 5 . Then, the application of the AHHS in patients with MELD less than 21 may further differentiate the patients who might benefit from treatment. Moreover, in this study, bilirubinostasis predicted (in nearly half of the patients) the development of bacterial infections, a severe complication associated to $\mathrm{AH}$. Clinicians must be aware to rule out infections in this subgroup of patients since sepsis is one of the main cause of death in severe $\mathrm{AH}$ and its presence can contraindicate corticosteroids.

As presented, we strongly recommend liver biopsy to confirm the diagnosis of $\mathrm{AH}$ and to discriminate patients with risk of infection and death without medical therapy. In our point of view, this method has been showed to be the best prognostic markers for $\mathrm{AH}$ in nowadays.

\section{Others Prognostic Factors}

Kasztelan et al [49] studying 116 patients demonstrated that alkaline phosphatase level higher than 1.5 times the upper limit of normal was an independent predictor of mortality of $\mathrm{AH}$ in 90 days. Moreover, this study demonstrated that two clinical parameters also had a significant prognostic value: all patients who died had ascites and $55 \%$ of them had also hepatic encephalopathy. Furthermore, the lack of ascites and encephalopathy was associated with $100 \%$ survival in 90 days [49].

The prognostic value of hepatic venous pressure gradient (HVPG) in patients with acute $A H$ was also evaluated. Rincon et al [50] studied 60 patients with severe $\mathrm{AH}$ and demonstrated that a HVPG of more than $22 \mathrm{mmHg}$ in patients with DF of greater 
than 32 was an independent predictor of in-hospital mortality [50]. On the other hand, a study done by Palaniyappan et al [39], was unable to demonstrate association between portal hypertension (HVPG $\geq$ $10 \mathrm{mmHg}$ ) with short or long survival in this group of patients [39].

The prognostic value of renal failure was assessed by Altamirano et al [51] in a study with 103 patients with $\mathrm{AH}$. Twenty-nine patients (28\%) developed acute kidney injury (AKI) during hospitalization. AKI was defined as an abrupt reduction (within $48 \mathrm{~h}$ ) in kidney function that resulted in an absolute increase of at least $0.3 \mathrm{mg} / \mathrm{dL}$ (or a $50 \%$ increase) in serum levels of creatinine from baseline (the AKI network [AKIN] criteria). In this study, the 90-day mortality was significantly higher among patients with AKI than those without (65\% vs $7 \%$ ). The most accurate predictors of AKI in this study were the presence of systemic inflammatory response syndrome, levels of bilirubin, and value of INR at admission. Furthermore, the AKIN criteria were more accurate than classical criteria for renal failure (serum creatinine $>1.5 \mathrm{mg} / \mathrm{dl}$ ) in predicting 90-day mortality [51].

In a recent study, serum levels of 25-hydroxyvitamin D were determined in 324 patients with ALD. Severe deficiency was significantly associated with more severe steatosis, fibrosis and $\mathrm{AH}$ [52].

\section{Conclusion}

$\mathrm{AH}$ is a severe complication among heavy drinkers and frequently results in poor short term prognosis. Various clinical scores are useful to differentiate patients with high mortality if not treated, these scores (DF, MELD, MELD Na, GAHS, ABIC) are similar in predicting the outcome. More recently, a histologic score including fibrosis, megamitochondria, neutrophil infiltration and bilirubinostasis showed promising results and can be recommend in a subset of patients submitted to liver biopsy.

\section{Author contributions}

Gabriela P Coral and Angelo A Mattos conceptualized the manuscript; Gabriela P Coral, Mariana A Pranke and Cristiane $\mathrm{V}$ Tovo reviewed the literature and wrote the manuscript; Angelo A Mattos and Cristiane $\mathrm{V}$ Tovo reviewed it critically for important intellectual content and all authors approved the final version to be published.

\section{Funding}

No.

\section{Competing and conflicting interests No.}

\section{References}

1. Lim SS, Vos T, Flaxman AD, Danaei G, Shibuya K, Amman M, et al. A comparative risk assessment of burden of disease and injury attributable to 67 risk factors and risk factor clusters in 21 regions, 1990-2010: a systematic analysis for the Global Burden of Disease Study 2010. Lancet 2012; 380: 2224-2260.

2. O'Shea RS, Dasarathy S, McCullough AJ. Practice Guideline Committee of the American Association for the Study of Liver Diseases; Practice Parameters Committee of the American College of Gastroenterology. Alcoholic liver disease. Hepatology 2010; 51: 307-328.

3. WHO. WHO Global status report on alcohol, [online], http:// www.who.int/mediacentre/factsheets/fs349/en/index.html (2011).

4. Rehm J, Samokhvalov AV, Shield KD. Global Burden of alcoholic liver diseases. J Hepatol 2013; 59: 160-168.

5. Gawryszewski VP, Monteiro MG. Mortality from diseases, conditions and injuries where alcohol is a necessary cause in the Americas, 2007-09. Addiction 2014; 109: 570-7.

6. Nader LA, Mattos AA, Bastos GAN. Burden of liver disease in Brazil. Liver Int 2014; 34: 844-849.

7. Crabb DW. Pathogenesis of alcoholic liver disease: newer mechanisms of injury. Keio J Med 1999; 48: 184-188.

8. Teli MR, Day CP, Burt AD, Bennett MK, James OF. Determinants of progression to cirrhosis or fibrosis in pure alcoholic fatty liver. Lancet 1995; 346: 987-990.

9. Orrego H, Blake JE, Blendis LM, Medline A. Prognosis of alcoholic cirrhosis in the presence and absence of alcoholic hepatitis. Gastroenterology 1987; 92: 208-214 
10. Lucey MR, Mathurin P, Morgan TR. Alcoholic hepatitis. N Engl J Med 2009; 360: 2758-2769.

11. Trabut JB, Plat A, Thepot $V$, Fontaine $H$, Vallet-Pichard A, et al. Influence of liver biopsy on abstinence in alcohol-dependent patients. Alcohol 2008; 43: 559-563.

12. Moreau R, Jalan R, Gines P, Pavesi M, Angeli P, Cordoba J, et al. CANONIC Study Investigators of the EASL-CLIF Consortium. Acute-on-Chronic Liver Failure Is a Distinct Syndrome That Develops in Patients With Acute Decompensation of Cirrhosis. Gastroenterology 2013; 144: 1426-1437.

13. Jaurigue MM, Cappell MS. Therapy for alcoholic liver disease. World J Gastroenterol 2014; 20: 2143-2158.

14. European Association for the Study of Liver. EASL clinical practical guidelines: management of alcoholic liver disease. J Hepatol 2012; 57: 399-420

15. Mathurin P, Mendenhall CL, Carithers RL, Ramond MJ, Maddrey WC, Garstide P, et al.Corticosteroids improve short-term survival in patients with severe alcoholic hepatitis $(\mathrm{AH})$ : individual data analysis of the last three randomized placebo controlled double blind trials of corticosteroids in severe AH. J Hepatol 2002; 36: 480-487

16. Mathurin P, O'Grady J, Carithers RL, Phillips M, Louvet A, Mendenhall $\mathrm{CL}$, et al. Corticosteroids improve short-term survival in patients with severe alcoholic hepatitis: meta-analysis of individual patient data. Gut 2011; 60: 255-260.

17. 17 Imperiale TF, McCullough AJ. Do corticosteroids reduce mortality from alcoholic hepatitis? A meta-analysis of the randomized trials. Ann Intern Med 1990; 113: 299-307.

18. Rambaldi A, Saconato HH, Christensen E, Thorlund K, Wetterslev J, Gluud C. Systematic review: glucocorticosteroids for alcoholic hepatitis--a Cochrane Hepato-Biliary Group systematic review with meta-analyses and trial sequential analyses of randomized clinical trials. Aliment Pharmacol Ther 2008; 27: 1167-1178.

19. Sidhu SS, Goyal O, Singla P, Gupta D, Sood A, Chhina RS, et al. Corticosteroid plus pentoxifylline is not better than corticosteroid alone for improving survival in severe alcoholic hepatitis (COPE trial). Dig Dis Sci 2012; 57: 1664-1671.

20. Mathurin P, Louvet A, Duhamel A, Nahon P, Carbonell N, Boursier J, et al. Prednisolone with vs without pentoxifylline and survival of patients with severe alcoholic hepatitis: a randomized clinical trial. JAMA 2013; 310: 1033-1041.

21. Lebrec D, Thabut D, Oberti F, Perarnau JM, Condat B, Barraud H, et al. Pentoxifylline does not decrease short term mortality but does reduce complications in patients with advanced cirrhosis. Gastroenterology 2010; 138: 1755-1762.

22. Thursz MR, Richardson $P$, Allison ME, Austin A, Bowers $M$. Steroids or Pentoxifylline for Alcoholic Hepatitis: Results of the STOPAH Trial. Late-Breaking Abstracts. Hepatology 2014; 60: 1267A.
23. Higuera-de la Tijera F, Servín-Caamaño Al, Cruz-Herrera J, Serralde-Zúñiga AE, Abdo-Francis JM, Gutiérrez-Reyes G, et al . Treatment with metadoxine and its impact on early mortality in patients with severe alcoholic hepatitis. Ann Hepatol 2014; 13: 343-52

24. Stickel F, Seitz HK. Update on the Management of Alcoholic Steatohepatitis. J Gastrointestin Liver Dis 2013; 22: 189-197.

25. Carithers RL, Herlong HF, Diehl AM, Shaw EW, Combes B, Fallon $\mathrm{HJ}$, et al. Methylprednisolone therapy in patients with severe alcoholic hepatitis. A randomized multicenter trial. Ann Intern Med 1989; 110: 685-690.

26. Kamath PS, Wiesner RH, Malinchoc M, Kremers W, Therneau $\mathrm{TM}$, Kosberg $\mathrm{CL}$ et al. A model to predict survival in patients with end-stage liver disease. Hepatology 2001; 33: 464-470.

27. Sheth $M$, Riggs $M$, Patel T. Utility of the Mayo End-Stage Liver Disease (MELD) score in assessing prognosis of patients with alcoholic hepatitis. BMC Gastroenterol 2002; 2: 2.

28. Dunn W, Jamil LH, Brown LS, Wiesner RH, Kim WR, Menon $\mathrm{KV}$, et al. MELD accurately predicts mortality in patients with alcoholic hepatitis. Hepatology 2005; 41: 353-358.

29. Srikureja W, Kyulo NL, Runyon BA, Hu KQ. MELD score is a better prognostic model than Child-Turcotte-Pugh score or Discriminant Function score in patients with alcoholic hepatitis. J Hepatol 2005; 42: 700-706.

30. Forrest EH, Evans CD, Stewart S, Phillips M, Oo YH, McAvoy NC. et al. Analysis of factors related to mortality in alcoholic hepatitis and the derivation and validation of the Glasgow alcoholic hepatitis score. Gut 2005; 54: 1174-9.

31. Forrest EH, Morris AJ, Stewart S, Phillips M, Oo YH, Fisher NC, et al. The Glasgow alcoholic hepatitis score identifies patients who may benefit from corticosteroids. Gut 2007; 56: 1743-1746.

32. Dominguez M, Rincón D, Abraldes JG, Miquel R, Colmenero J, Bellot $\mathrm{P}$, et al. A New scoring system for prognostic stratification of patients with alcoholic hepatitis. Am J Gastroenterol 2008; 103: 2747-56.

33. Louvet A, Naveau S, Abdelnour M, Ramond MJ, Diaz E, Dharancy $S$, et al. The Lille model: a new tool for thepapeutic strategy in patients with severe alcoholic hepatitis treated with steroids. Hepatology 2007; 45: 1348-54.

34. Mathurin P, Abdelnour M, Ramond MJ, Carbonell N, Fartoux L, Serfaty $L$, et al. Early change in bilirubin levels is an important prognostic factor in severe alcoholic hepatitis treated with prednisolone. Hepatology 2003; 38: 1363-9.

35. Maddrey WC, Boitnott JK, Bedine MS, Weber FL Jr, Mezey E, White RI Jr. Corticosteroid therapy of alcoholic hepatitis. Gastroenterology 1978; 75: 193-199.

36. Vaa BE, Asrani SK, Dunn W, Kamath PS, Shah VH. Influence of Serum Sodium on MELD-Based Survival Prediction in Alcoholic Hepatitis. Mayo Clin Proc 2011; 86: 37-42.

37. Morris JM, Forrest EH. Bilirubin response to corticosteroids in severe alcoholic hepatitis. Eur J Gastroenterol Hepatol 2005; 17: 759-62. 
38. Sandahl TD, Jepsen $P$, Ott $P$, Vilstrup $H$. Validation of prognostic scores for clinical use in patients with alcoholic hepatitis. Scand J Gastroenterol 2011; 46: 1127-32.

39. Palaniyappan N, Subramanian $V$, Ramappa $V$, Ryder SD, Kaye P, Aithal GP. The Utility of Scoring Systems in Predicting Early and Late Mortality in Alcoholic Hepatitis: Whose Score Is It Anyway? International J Hepatol 2012; 2012: 624675.

40. Lafferty H, Stanley AJ, Forrest EH. The management of alcoholic hepatitis: a prospective comparison of scoring systems. Aliment Pharmacol Ther 2013; 38: 603-10.

41. Papastergiou $V$, Tsochatzis EA, Pieri G, Thalassinos E, Dhar A, Bruno $S$, et al. Nine scoring models for short-term mortality in alcoholic hepatitis: cross-validation in a biopsy-proven cohort. Aliment Pharmacol Ther 2014; 39: 721-32.

42. Cuthbert JA, Arslanlar S, Yepuri J, Montrose M, Ahn CW. Predicting short-term mortality and long-term survival for hospitalized US patients with alcoholic hepatitis. Dig Dis Sci 2014; 597: 1594-602.

43. Lee M, Kim W, Choi Y, Kim S, Kim D, Yu SJ, et al. Spontaneous evolution in bilirubin levels predicts liver-related mortality in patients with alcoholic hepatitis. PLoS One 2014; 9: e100870.

44. Hall PD. Pathological spectrum of alcoholic liver disease. Alcohol Alcohol Suppl 1994; 2: 303-313.

45. Lefkowitch JH. Morphology of alcoholic liver disease. Clin Liver Dis 2005; 9: 37-53.

46. Mathurin $P$, Beuzin $F$, Louvet $A$, Carrié-Ganne N, Balian A, Trinchet JC, et al. Fibrosis progression occurs in a subgroup of heavy drinkers with typical histological features. Aliment Pharmacol Ther 2007; 25: 1047-1054.

47. Spahr L, Rubbia-Brandt L, Genevay M, Hadengue A, Giostra E. Early liver biopsy, intraparenchymal cholestasis, and prognosis in patients with alcoholic steatohepatitis. BMC Gastroenterol 2011; 11: 115.

48. Altamirano J, Miquel R, Katoonizadeh A, Abraldes JG, DuarteRojo A, Louvet A, et al. A Histologic Scoring System for Prognosis of Patients With Alcoholic Hepatitis. Gastroenterology 2014; 146: 1231-1239.

49. Kasztelan-Szczerbinska B, Slomka M, Celinski K, Szczerbinski M. Alkaline phosphatase: the next independent predictor of the poor 90-day outcome in alcoholic hepatitis. Biomed Res Int 2013; 2013: 614081
50. Rincon D, Lo lacono O, Ripoll C, Gomez-Camarero J, Salcedo $M$, et al (2007) Prognostic value of hepatic venous pressure gradient for in-hospital mortality of patients with severe acute alcoholic hepatitis. Aliment Pharmacol Ther 25: 841-8.

51. Altamirano J, Fagundes $C$, Dominguez M, García E, Michelena J, et al (2012) Acute kidney injury is an early predictor of mortality for patients with alcoholic hepatitis. Clin Gastroenterol Hepatol 10: 65-71.

52. Trépo $E$, Ouziel $R$, Pradat $P$, Momozawa $Y$, Quertinmont $E$, Gustot T, et al. Marked 25-hydroxyvitamin D deficiency is associated with poor prognosis in patients with alcoholic liver disease. J Hepatol 2013; 59: 344-350.

\section{Comment on this article:}

\section{(f) $[$ in $8+\boldsymbol{S} P$}

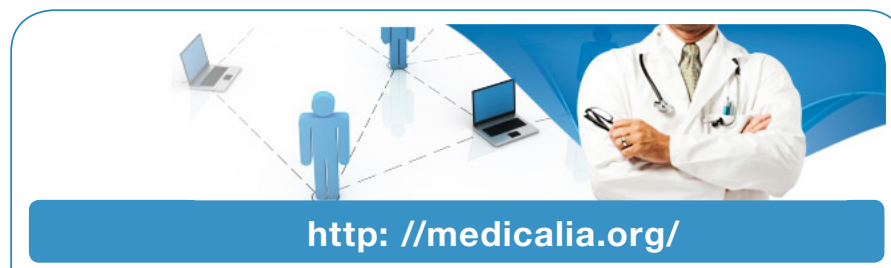

Where Doctors exchange clinical experiences, review their cases and share clinical knowledge. You can also access lots of medical publications for free. Join Now!

\section{Publish with iMedPub}

http: //www.imed.pub

International Archives of Medicine is an open access journal publishing articles encompassing all aspects of medical science and clinical practice. IAM is considered a megajournal with independent sections on all areas of medicine. IAM is a really international journal with authors and board members from all around the world. The journal is widely indexed and classified Q1 in category Medicine. 